Passions of the Tongue 
Studies on the History of Society and Culture

Victoria E. Bonnell and Lynn Hunt, Editors

I. Politics, Culture, and Class in the French Revolution, by Lynn Hunt

2. The People of Paris: An Essay in Popular Culture in the Eighteenth Century, by Daniel Roche

3. Pont-St-Pierre, 1398-1789: Lordship, Community, and Capitalism in Early Modern France, by Jonathan Dewald

4. The Wedding of the Dead: Ritual, Poetics, and Popular Culture in Transylvania, by Gail Kligman

5. Students, Professors, and the State in Tsarist Russia, by Samuel D. Kassow

6. The New Cultural History, edited by Lynn Hunt

7. Art Nouveau in Fin-de-Siècle France: Politics, Psychology, and Style, by Debora L. Silverman

8. Histories of a Plague Year: The Social and the Imaginary in Baroque Florence, by Giulia Calvi

9. Culture of the Future: The Proletkult Movement in Revolutionary Russia, by Lynn Mally

Io. Bread and Authority in Russia, 1914-1921, by Lars T. Lih

II. Territories of Grace: Cultural Change in the Seventeenth-Century Diocese of Grenoble, by Keith P. Luria

I2. Publishing and Cultural Politics in Revolutionary Paris, $1789-1810$, by Carla Hesse

13. Limited Livelihoods: Gender and Class in Nineteenth-Century England, by Sonya O. Rose

I4. Moral Communities: The Culture of Class Relations in the Russian Printing Industry, 1867-1907, by Mark Steinberg

I 5. Bolshevik Festivals, 1917-1920, by James von Geldern

16. Venice's Hidden Enemies: Italian Heretics in a Renaissance City, by John Martin

17. Wondrous in His Saints: Counter-Reformation Propaganda in Bavaria, by Philip M. Soergel

I8. Private Lives and Public Affairs: The Causes Célebres of Prerevolutionary France, by Sarah Maza

19. Hooliganism: Crime, Culture, and Power in St. Petersburg, I900-19I4, by Joan Neuberger

20. Possessing Nature: Museums, Collecting, and Scientific Culture in Early Modern Italy, by Paula Findlen 
21. Listening in Paris: A Cultural History, by James H. Johnson

22. The Fabrication of Labor: Germany and Britain, $1640-1914$, by Richard Biernacki

23. The Struggle for the Breeches: Gender and the Making of the British Working Class, by Anna Clark

24. Taste and Power: Furnishing Modern France, by Leora Auslander

25. Cholera in Post-Revolutionary Paris: A Cultural History, by Catherine J. Kudlick

26. The Women of Paris and Their French Revolution, by Dominique Godineau

27. Iconography of Power: Soviet Political Posters under Lenin and Stalin, by Victoria E. Bonnell

28. Aestheticized Politics: Fascism in Italy, 1919-1940, by Simonetta Falasca-Zamponi

29. Passions of the Tongue: Language Devotion in Tamil India, 1891-1970, by Sumathi Ramaswamy 
A

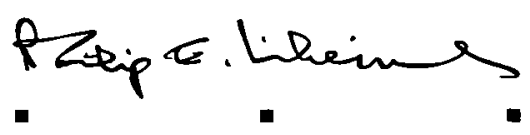

Book

The Philip E. Lilienthal imprint honors special books

in commemoration of a man whose work at the University of California Press from 1954 to 1979

was marked by dedication to young authors and to high standards in the field of Asian Studies. Friends, family, authors, and foundations have together endowed the Lilienthal Fund, which enables the Press

to publish under this imprint selected books in a way that reflects the taste and judgment of a great and beloved editor. 


\section{Passions of the Tongue}

Language Devotion in

Tamil India, I891-1970

\section{Sumathi Ramaswamy}

UNIVERSITY OF CALIFORNIA PRESS

Berkeley · Los Angeles · London 
University of California Press

Berkeley and Los Angeles, California

University of California Press, Ltd.

London, England

C) 1997 by

The Regents of the University of California

Library of Congress Cataloging-in-Publication Data

Ramaswamy, Sumathi.

Passions of the tongue : language devotion in Tamil India, I891-1970 / Sumathi Ramaswamy.

p. $\quad \mathrm{cm}$.- (Studies on the history of society and culture ; 29)

Includes bibliographical references and index.

ISBN 0-520-20804-8 (cloth : alk. paper).-

ISBN 0-520-20805-6 (pbk. : alk. paper)

I. Ramaswamy, Sumathi-Knowledge-

Dravidian languages. 2. Language and lan-

guages-Study and teaching-United States.

3. Critical pedagogy-United States. 4. IndiaLanguages-Political aspects. 5. Language and culture-India-History. 6. Nationalism-

India-History. 7. India-History-British occupation, 1765-r947-Historiography.

I. Series.

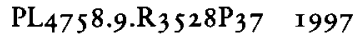

494'.8 I I'0954-dc2I

$96-5244 I$

Printed in the United States of America

$\begin{array}{lllllllll}9 & 8 & 7 & 6 & 5 & 4 & 3 & 2 & \text { I }\end{array}$

The paper used in this publication is both acid-free and totally chlorine-free (TCF). It meets the minimum requirements of American Standard for Information Sciences-Permanence of Paper for Printed Library Materials, ANSI Z39.48-1984. 
For appa, amma, and Rich 
இருந்தம்ழே யுற்னா லிருந்தே ரிமையோர்

விருந்தமிழ்த மென்றாலும் Cேண்டேன்.

$$
\text { தமிழ் வீடிதூது }
$$

பொன் ஜும் புவியும் புதை பொருளும் யான் வேண்டேன் துன் னு சுடர்விழயயார்த் தமிழணங்கே! நின்னடிக்க்ழ பன் இுந் துமிழ்ப் பஜுவற் பாடிடவுங் கேட்டிடவு மின் னும் மென வெழூஞ்ச ரிதயமே வேண்டுவனால்!

இரா. வாசுதேவ சர்மா

எங்கள் உடல் பொருள் ஆவியெல்லாம் - எங்கள் இன்பத் கமிழ் மொழக்கே தருவோம்! மங்கை ஒருத்தி தரும் சுகமும் - எங்கள் மாத்தடிழ்க் கீடில்லை என்றுறைப்போம்!

பாரதிதாசன் கமிழ்ப் போரே எனது வாழ்க்கைப் போர். இஸ்குவனார் 
O preeminent Tamil! I exist because of you!

Even the ambrosia of the celestials, I do not desire!

Tamil Viṭutūtu, $c$. I 7 th century

I want neither gold nor the earth nor hidden treasures,

O bright-eyed Goddess Tamil!

I pray for a good heart that longs to sing and hear sweet

Tamil song,

at your feet!

Vasudeva Sharma, 1928

Our bodies, our wealth, our very breath,

We will surrender to our sweet Tamil!

Even the pleasures woman alone gives do not compare to our great Tamil,

We will declare!

Bharatidasan, 1938

The battle for Tamil is the battle of my life.

Ilakuvanar, $x 97$ I 
